\title{
High-Pressure Phase Stability and Superconductivity of Pnictogen Hydrides and Chemical Trends for Compressed Hydrides
}

Yuhao Fu, ${ }^{\dagger}$ Xiangpo Du, ${ }^{\ddagger}$ Lijun Zhang, ${ }^{, \dagger}{ }^{\dagger}$ Feng Peng, ${ }^{\ddagger}$ Miao Zhang, ${ }^{\ddagger}$ Chris J. Pickard, ${ }^{\mathbb{I}}$ Richard J. Needs, ${ }^{\S}$ David J. Singh, ${ }^{*},{ }^{\dagger}, \|$ Weitao Zheng, ${ }^{\dagger}$ and Yanming Ma, ${ }^{*}$

${ }^{\dagger}$ College of Materials Science and Engineering and Key Laboratory of Automobile Materials of MOE and ${ }^{\ddagger}$ State Key Laboratory of Superhard Materials, Jilin University, Changchun 130012, China

${ }^{\mathbb{T}}$ Department of Materials Science \& Metallurgy, University of Cambridge, 27 Charles Babbage Road, Cambridge CB3 0FS, United Kingdom

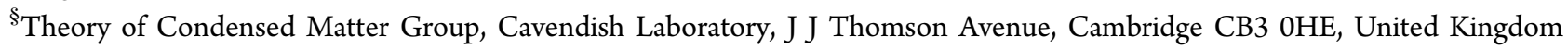

"Department of Physics and Astronomy, University of Missouri, Columbia, Missouri 65211-7010, United States

\section{Supporting Information}

ABSTRACT: The recent breakthrough discovery of unprecedentedly high temperature superconductivity of $203 \mathrm{~K}$ in compressed sulfur hydrides has stimulated significant interest in finding new hydrogencontaining superconductors and elucidating the physical and chemical principles that govern these materials and their superconductivity. Here we report the prediction of high temperature superconductivity in the family of pnictogen hydrides using first-principles calculations in combination with global optimization structure searching methods. The hitherto unknown high-pressure phase diagrams of binary hydrides formed by the pnictogens of phosphorus, arsenic, and antimony are explored, stable structures are identified, and their electronic, vibrational, and superconducting properties are investigated. We predict that $\mathrm{SbH}_{4}$ and $\mathrm{AsH}_{8}$ are high-temperature superconductors at megabar pressures,

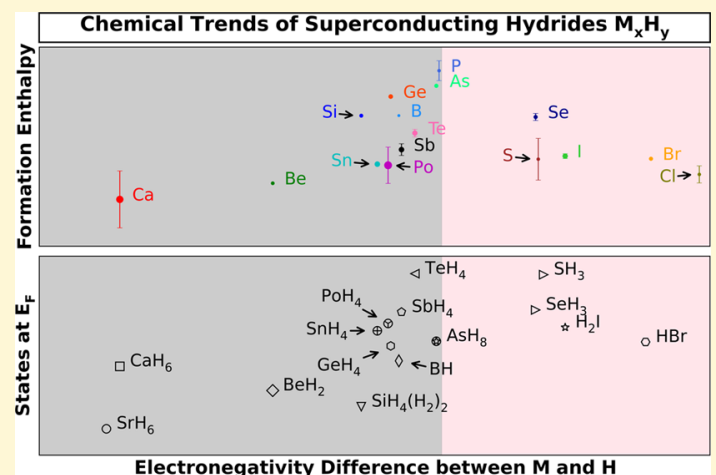
with critical temperatures in excess of $100 \mathrm{~K}$. The highly symmetrical hexagonal $\mathrm{SbH}_{4}$ phase is predicted to be stabilized above about $150 \mathrm{GPa}$, which is readily achievable in diamond anvil cell experiments. We find that all phosphorus hydrides are metastable with respect to decomposition into the elements within the pressure range studied. Trends based on our results and data in the literature reveal a connection between the high-pressure behaviors and ambient-pressure chemical quantities which provides insight into understanding which elements may form hydrogen-rich high-temperature superconducting phases at high pressures.

\section{INTRODUCTION}

Superconductivity, especially at high temperatures, continues to offer surprises both from experimental discoveries and theoretical insights often stimulated by these experiments. These findings include the Fe-based superconductors ${ }^{1}$ which have provided a large body of theoretical work on spinfluctuation pairing, nematicity, and multiorbital physics ${ }^{2-4}$ and recent reports of superconductivity in sulfur hydrides at pressures $\sim 200 \mathrm{GPa}$ with a $T_{c}$ of $203 \mathrm{~K}^{5,6}$ Superconducting sulfur hydrides show a strong isotope effect when deuterium is substituted for hydrogen, as expected for electron-phononbased superconductivity. ${ }^{5}$ This breakthrough observation has led to debate on the superconducting mechanism, including discussion of the limits of standard Migdal-Eliashberg electron-phonon coupling (EPC) theory, ${ }^{7-9}$ the role of strong covalent bonding, ${ }^{10,11}$ the effects of anharmonicity on EPC, ${ }^{12}$ etc. These proposals, together with the pioneering work of Ashcroft and co-workers on predicted high-temperature superconductivity in solid hydrogen ${ }^{13}$ and in hydrogen-rich hydrides, ${ }^{14,15}$ may guide research toward the discovery of new high-temperature superconductors based on materials containing light elements.

High-temperature superconductors can be divided into two classes: unconventional superconductors characterized by nonphonon primary pairing interactions, normally leading to nonstandard pairing states, and conventional superconductors, with dominant electron-phonon pairing, and s-wave (or mainly s-wave for noncentrosymmetric cases) pairing states. In the latter case, methods exist for reliably evaluating superconducting properties and analyzing the superconductivity, e.g., through calculating the Eliashberg EPC spectral function $\alpha^{2} \mathrm{~F}(\omega)$. A general consensus has been reached that the recently discovered superconducting sulfur hydrides belong to this category. ${ }^{5,10,16-19}$ The experimental discovery was

Received: December 1, 2015

Revised: February 14, 2016

Published: February 17, 2016 
motivated by a theoretical prediction of high- $T_{c}$ superconductivity in compressed solid $\mathrm{H}_{2} \mathrm{~S}$ by some of us. ${ }^{16}$ Two superconducting states are observed in $\mathrm{H}_{2} \mathrm{~S}$ : samples prepared at low temperature have a maximum $T_{c}$ of $\sim 150 \mathrm{~K}$ at $200 \mathrm{GPa}$, while the superconductivity at $203 \mathrm{~K}$ arises from samples prepared via annealing at temperatures above $220 \mathrm{~K}$. The latter phase originates from the decomposition of $\mathrm{H}_{2} \mathrm{~S}$ into $\mathrm{H}_{3} \mathrm{~S}$. ${ }^{6,12,17}$

Theoretical predictions of high-temperature superconductivity rely on knowledge of the stable structures of the system. Global optimization structure searching methods ${ }^{20}$ can identify previously unknown ground-state structures such as sulfur hydrides at high pressures. ${ }^{12,16,17,21}$ Calculations based on density functional theory (DFT) allow an accurate energetic evaluation of the structures found within the potential energy landscape and an assessment of their potential for superconductivity. The experimental discovery of high-temperature superconducting sulfur hydrides demonstrates the predictive power of DFT-based structure searching and EPC calculations and suggests that more superconductors could be discovered using this approach. The role of theory is especially important since under such extreme conditions sample synthesis and in situ measurements of properties are usually challenging. Recently, the sister systems of selenium and tellurium hydrides were predicted to exhibit high- $T_{c}$ superconductivity in hydrogen-rich compounds stabilized above $100 \mathrm{GPa}^{22,23}$ These proposals await experimental testing.

Here we explore the thermodynamic stability of pnictogen hydrides of $\mathrm{P}, \mathrm{As}$, and $\mathrm{Sb}$ at high pressures and their superconducting properties. This investigation is in part motivated by the fact that the strength of the covalent $\mathrm{H}-\mathrm{P}$ bond (with a bond energy of $322 \mathrm{~kJ} / \mathrm{mol}$ ) is rather similar to that of the $\mathrm{H}-\mathrm{S}$ bond (bond energy of $363 \mathrm{~kJ} / \mathrm{mol}$ ) in the molecular gas phase. It is therefore reasonable to conjecture, within the scenario that strong covalent bonding favors superconductivity, ${ }^{10,11}$ that phosphorus hydrides containing strong covalent bonds to $\mathrm{H}$ atoms and becoming metallic under compression might exhibit similarly strong EPC and potentially high- $T_{c}$ superconductivity. To the best of our knowledge, solid pnictogen hydrides, even at ambient conditions, have only rarely been studied, ${ }^{24-27}$ although the corresponding molecular gases (e.g., $\mathrm{PH}_{3}, \mathrm{P}_{2} \mathrm{H}_{4}, \mathrm{AsH}_{3}$, and $\mathrm{SbH}_{3}$ ) are well-known in chemistry.

We find that thermodynamic stability of the gas molecules $\left(\mathrm{PH}_{3}>\mathrm{AsH}_{3}>\mathrm{SbH}_{3}\right)$ is reversed at high pressures, resulting in decomposition enthalpies that decrease from $\mathrm{P}$, As to $\mathrm{Sb}$ hydrides. Except for the molecular solid phases stabilized by weak van der Waals interactions at low pressures, ${ }^{24-27} \mathrm{P}$ hydrides are found to exhibit thermodynamic instability to decomposition into the elements under pressures up to 400 GPa. For As hydrides, stable compounds emerge only above $300 \mathrm{GPa}$. Sb hydrides are the most easily stabilized compounds above $150 \mathrm{GPa}$, which is readily achievable in diamond anvil cells. We have identified two stable hydrogen-rich compounds, $\mathrm{SbH}_{4}$ and $\mathrm{AsH}_{8}$, as possible high-temperature superconductors with a $T_{c}$ of $102 \mathrm{~K}$ at $150 \mathrm{GPa}$ and $141 \mathrm{~K}$ at $350 \mathrm{GPa}$, respectively. Based on analysis of current and prior theoretical work on other binary hydrides, we have established a connection between ambient-pressure chemical properties and energetic stability, structural features, superconducting properties, etc. of hydrides upon pressures. The trends implied by this connection may help in discovering new binary and multinary high-pressure hydrogen-rich superconductors, since it does not require knowledge of the very different chemistries of elements at high pressures. Using trends in behavior to help in discovering superconductors has long been recognized, starting with the rules set out by Matthias. ${ }^{28,29}$ Finally, we mention the puzzling fact that although there have been more than a dozen predictions of high-temperature superconductivity in compressed hydrogen-rich compounds, ${ }^{16,17,19,22,23,30-39}$ sulfur hydride is the only system to date that has been demonstrated to exhibit a higher superconducting temperature than the record of $164 \mathrm{~K}$ set by cuprates. ${ }^{40}$ The second higher$T_{c}$ material identified in experiments is silicon hydride with $T_{c}=$ $17 \mathrm{~K}$ around $100 \mathrm{GPa}^{41}$ although the origin of the superconductivity is still under debate (likely from platinum hydride). ${ }^{42,43}$ The chemical and physical properties of sulfur hydride that make it the highest- $T_{c}$ superconductor found so far are not yet fully understood. Here we provide insight into this puzzle based on trends derived from data for a wide range of high-pressure hydrides.

\section{COMPUTATIONAL APPROACHES}

Our investigation consists of the determination of stable stoichiometries and crystalline structures and calculation of their superconducting and related properties. The hitherto unknown high-pressure phase diagrams $($ at $0 \mathrm{~K})$ of pnictogen $(\mathrm{X})$ hydrides were explored by performing very extensive structure searches for a set of stoichiometries $\mathrm{X}_{n} \mathrm{H}_{m}$, and the most stable structure and low-lying enthalpy metastable structures were identified for each $\mathrm{X}_{n} \mathrm{H}_{m}$. These calculations were performed using two leading codes for firstprinciples structure prediction, CALYPSO ${ }^{44,45}$ and AIRSS. ${ }^{46,47}$ The energetically stable stoichiometries and the most stable structure at each stoichiometry obtained from the two searching methods are in agreement. Over 100 stoichiometries were searched, and a total of about 100,000 structures were relaxed by minimizing the total enthalpy within the $\mathrm{P} / \mathrm{H}$, As $/ \mathrm{H}$, and $\mathrm{Sb} / \mathrm{H}$ systems.

DFT calculations were performed using the VASP code ${ }^{48}$ with projected-augmented-wave (PAW) ${ }^{49}$ potentials and the CASTEP code with ultrasoft pseudopotentials. The $1 s(\mathrm{H}), 3 s$ and $3 p(\mathrm{P}), 4 s$ and $4 p$ $(\mathrm{As})$, and $5 s$ and $5 p(\mathrm{Sb})$ electrons were treated explicitly as valence electrons. We used the generalized gradient approximation exchangecorrelation functional of Perdew, Burke, and Ernzerhof. ${ }^{50}$ Medium quality computational parameters were used when evaluating the enthalpies of structures during the searches. The low-lying enthalpy structures were then further optimized using the PAW method and more accurate computational parameters consisting of kinetic energy cutoff energies of $650 \mathrm{eV}(\mathrm{P} / \mathrm{H}$ and $\mathrm{As} / \mathrm{H})$ and $510 \mathrm{eV}(\mathrm{Sb} / \mathrm{H})$ and $\mathrm{k}-$ point meshes of spacing $2 \pi \times 0.03 \AA^{-1}$ or less. We checked the energy convergence with respect to these parameters and found convergence of the enthalpy differences to better than $1 \mathrm{meV} /$ atom level.

We calculated the phonon dispersion relations and EPC using linear response theory as implemented in the Quantum ESPRESSO package. $^{51}$ These calculations were performed using norm-conserving pseudopotentials and a kinetic energy cutoff of $100 \mathrm{Ry}$. For reliable evaluation of the double-delta function integrals in the EPC calculations, dense k-point meshes with a spacing of about $2 \pi \times$ $0.015 \AA^{-1}$ were used, in combination with the Methfessel-Paxton broadening scheme ${ }^{52}$ with a broadening parameter of 0.05 Ry. For the Brillouin sampling of phonon momentum, a q-point mesh with a grid spacing of $\sim 2 \pi \times 0.06 \AA^{-1}$ was adopted.

\section{RESULTS AND DISCUSSION}

3.1. Phase Stabilities and Stable Crystalline Structures of Pnictogen Hydrides at High Pressures. Since H-rich compounds are potentially more favorable for high-temperature superconductivity, ${ }^{14}$ we focused our CALYPSO searches on the $\mathrm{X}_{n} \mathrm{H}_{m}(n=1-3$ and $m=1-8)$ stoichiometries with higher $\mathrm{H}$ contents $(m \geq n)$ containing up to 4 formula units per simulation cell. More diverse stoichiometries (with $m$ up to 13) 
were considered in the AIRSS searches. The main results from these cross-checking search calculations are depicted in the hull diagrams of Figure 1, which each contain data for pressures of

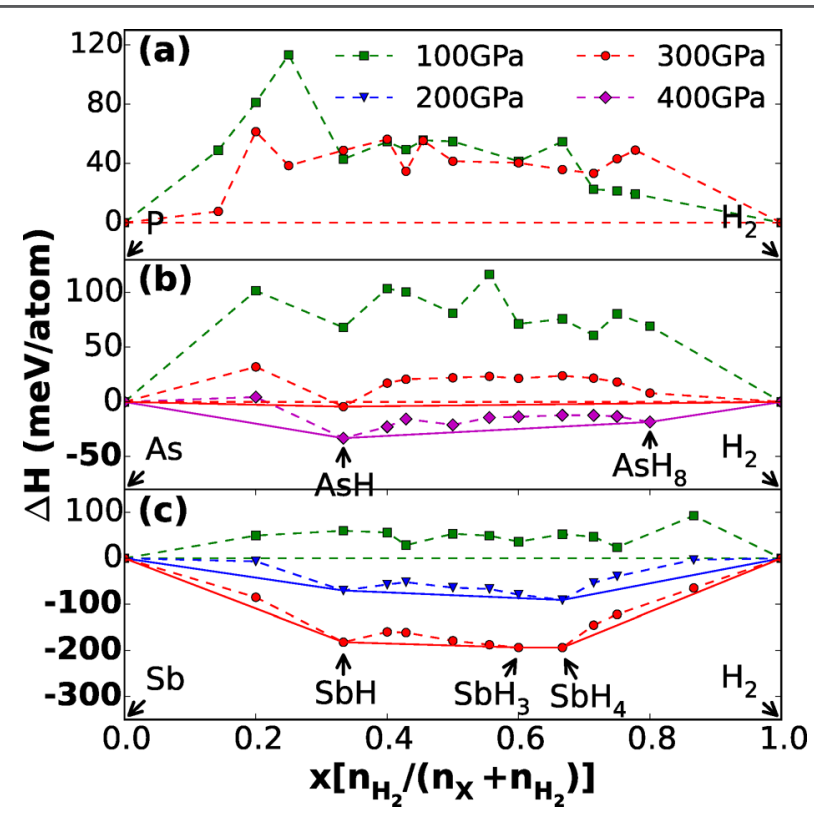

Figure 1. Calculated formation enthalpies $\Delta H$ (in $\mathrm{meV} /$ atom) of various pnictogen hydrides with respect to decomposition into the elemental solids at 100 (squares), 200 (triangles), 300 (circles), and 400 (diamond) GPa, respectively. The data for each stoichiometry corresponds to the lowest enthalpy structures from the searches. The structures of phases VI $(P m \overline{3} m)$ and VIII $(\operatorname{Im} \overline{3} m)$ of $\mathrm{P},{ }^{53} \operatorname{IV}(\operatorname{Im} \overline{3} m)$ of $\mathrm{As}^{53}$ III $(\operatorname{Im} \overline{3} m)$ of $S b,{ }^{53}$ and the $P 6_{3} m$ and $C 2 / c$ structures of solid $\mathrm{H}_{2}{ }^{54}$ were used for evaluating $\Delta H$.

100-300 GPa. For As hydrides (Figure 1b), searches at the higher pressure of $400 \mathrm{GPa}$ were performed to demonstrate the stability of the $\mathrm{AsH}$ and $\mathrm{AsH}_{8}$ structures above $300 \mathrm{GPa}$. Only the lowest enthalpy structure at each stoichiometry studied is shown.

We find a surprising trend in that at high pressures the thermodynamic stability of pnictogen hydrides increases with the atomic number of the pnictogen. The P hydrides (Figure 1a) are found to be unstable against decomposition into the elements, and pressure does not have a noticeable effect on their stability. The predicted lowest-energy structures of several metastable stoichiometries are shown in Supplementary Figure $\mathrm{S} 1$ and Table S1. As and Sb hydrides (Figure $1 \mathrm{~b}$ and $1 \mathrm{c}$ ) show a clear tendency to be stabilized by increasing pressure; for As hydrides, a single stoichiometry stable against elemental decomposition into the elements emerges at $300 \mathrm{GPa}$, while for $\mathrm{Sb}$ hydrides, all the stoichiometries become stable above $200 \mathrm{GPa}$. These results indicate that the relative stabilities of the pnictogen hydrides obtained from the known chemical bonding strengths at ambient conditions $(P>\mathrm{As}>\mathrm{Sb})$ are opposite to those found at high pressures $(P<\mathrm{As}<\mathrm{Sb})$. We find two stable binary As hydrides on the convex hull at 400 $\mathrm{GPa}, \mathrm{AsH}$, and $\mathrm{AsH}_{8}$. We find three compounds on the convex hull for Sb hydrides at $300 \mathrm{GPa}$ and two at $200 \mathrm{GPa}$. These are $\mathrm{SbH}, \mathrm{SbH}_{3}$ (at $300 \mathrm{GPa}$ only), and $\mathrm{SbH}_{4}$. Note that the much lower formation enthalpies of $\mathrm{Sb}$ hydrides $(\sim 200 \mathrm{meV} /$ atom more stable) demonstrate their high stability under compression. We evaluated the effect of zero point energy (ZPE) on the phase stability by calculating harmonic phonon spectra for $\mathrm{Sb}$ hydrides, as shown in Supplementary Figure S2. Inclusion of the ZPE gives rise to a negligible change in the convex hulls.

The structures of the predicted stable compounds are shown in Figure 2a-e (see Supplementary Table S2 for detailed

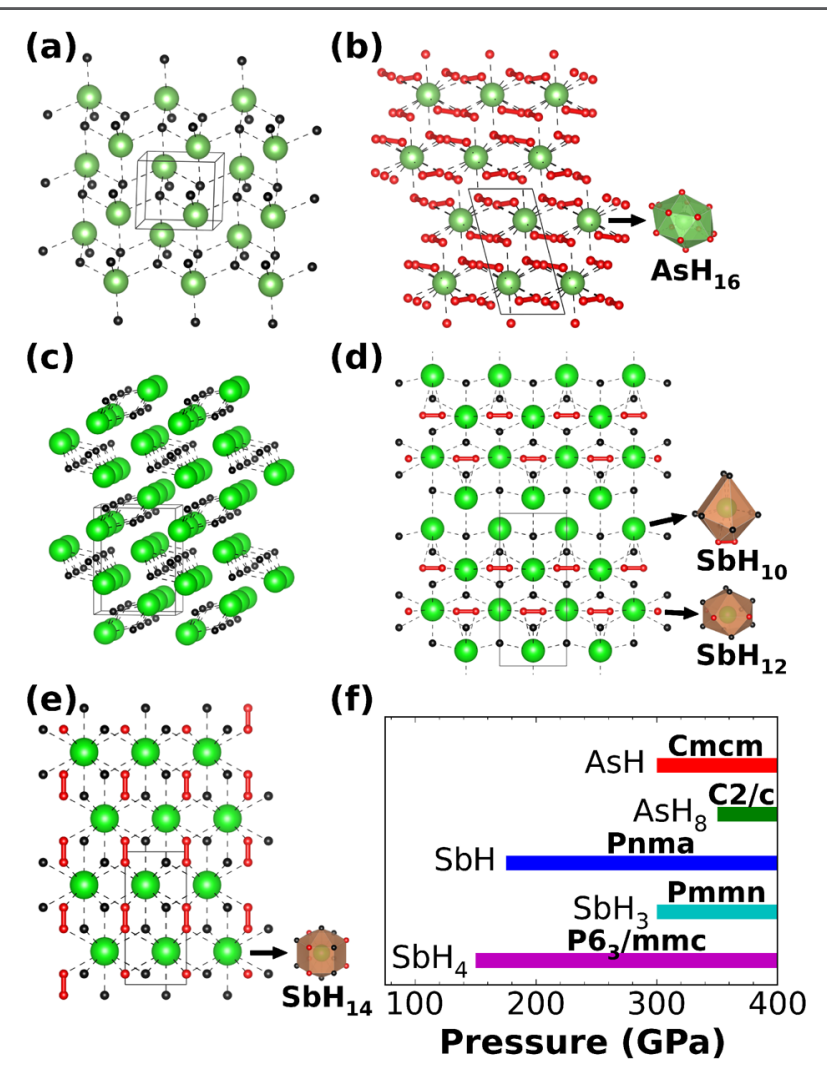

Figure 2. Structures of the predicted stable pnictogen hydrides: (a) $\mathrm{AsH}$ (space group $\mathrm{Cmcm}$ ), (b) $\mathrm{AsH}_{8}(\mathrm{C} 2 / \mathrm{c}),(\mathrm{c}) \mathrm{SbH}$ (Pnma), (d) $\mathrm{SbH}_{3}(\mathrm{Pmmn}) ;\left(\right.$ e) $\mathrm{SbH}_{4}\left(\mathrm{~Pb}_{3} / m m c\right)$. The As/Sb-H bonds are depicted by dashed lines and $\mathrm{H}-\mathrm{H}$ covalent bonds as red sticks. The corresponding stable pressure range of each compound is shown in (f).

structural information), and the pressure ranges within which they are stable are depicted in Figure $2 \mathrm{f}$. The stable structures of the compounds remain unchanged throughout their stable pressure ranges. The AsH hydride (Figure 2a) adopts a structure of $\mathrm{Cmcm}$ symmetry which is stable above $300 \mathrm{GPa}$. It consists of a three-dimensional As- $\mathrm{H}$ network in which both As and $\mathrm{H}$ atoms are approximately 5 -fold coordinated. The lowest enthalpy structure of $\mathrm{AsH}_{8}$ (Figure 2b) of $\mathrm{C} 2 / \mathrm{c}$ symmetry is stabilized above $350 \mathrm{GPa}$. It is formed from the primary motifs of irregular $\mathrm{AsH}_{16}$ polyhedra, connected with each other in a three-dimensional network. Each corner $\mathrm{H}$ atom of the $\mathrm{AsH}_{16}$ polyhedron is linked to another $\mathrm{H}$ from the adjacent polyhedron. The short contact between two $\mathrm{H}$ atoms leads to formation of an intriguing quasi-molecular $\mathrm{H}_{2}$-unit with a bond length of $\sim 0.8-0.9 \AA$. The formation of such $\mathrm{H}_{2}$-units in compressed hydrides is also seen in systems with $\mathrm{Si}, \mathrm{Ge}, \mathrm{Sn}$, $\mathrm{Li}, \mathrm{Ca}, \mathrm{Te},^{23,30,32,33,55,56}$ etc.

The energetically favored structure of $\mathrm{SbH}$ (Figure 2c, stable above $175 \mathrm{GPa}$ ) has space group Pnma and is composed of chain-like $\mathrm{Sb}-\mathrm{H}$ motifs with each $\mathrm{Sb}$ coordinated by three $\mathrm{H}$ atoms. The H-rich compounds $\mathrm{SbH}_{3}$ (Figure 2d) and $\mathrm{SbH}_{4}$ (Figure 2e) have $P m m n$ and $\mathrm{PG}_{3} / m m c$ symmetries, respectively. While the former is marginally stable (very close to the convex hull formed by $\mathrm{SbH}$ and $\mathrm{SbH}_{3}$ in Figure 1c) at $300 \mathrm{GPa}$, the 
latter is robustly stable above $150 \mathrm{GPa}$. $\mathrm{SbH}_{3}$ is composed of irregular polyhedral $\mathrm{SbH}_{10}$ and $\mathrm{SbH}_{12}$ motifs which form its three-dimensional topology. Bridging the adjacent $\mathrm{SbH}_{12}$ motifs in a two-dimensional fashion gives rise to quasi-molecular $\mathrm{H}_{2}$ units (in red) with a rather long bond length of $\sim 0.91 \AA$, compared with the molecular $\mathrm{H}_{2}$ bond length of $0.74 \AA . \mathrm{SbH}_{4}$ is a highly symmetrical hexagonal compound that consists of $\mathrm{SbH}_{14}$ octadecahedra connected via shared $\mathrm{H}$ atoms at the corners to form a three-dimensional network. There are two types of $\mathrm{H}$ atoms occupying the $4 e$ (black) and $4 f$ (red) Wyckoff sites, respectively. Each $4 e \mathrm{H}$ atom is coordinated by three $\mathrm{Sb}$ atoms, and the $4 f \mathrm{H}$ atom, while coordinated by two $\mathrm{Sb}$ atoms, has close contact with another $4 f \mathrm{H}$ atom, forming quasi-molecular $\mathrm{H}_{2}$-units with a bond length of $\sim 0.83 \AA . \mathrm{SbH}_{4}$ resembles the $\mathrm{TeH}_{4}$ compound with the same stoichiometry which adopts the $R \overline{3} m$ phase. $^{23}$

A Bader charge density analysis ${ }^{57}$ shows substantial charge transfer from As/Sb to H (see Supplementary Table S3). This indicates that the $\mathrm{As} / \mathrm{Sb}-\mathrm{H}$ bonds have substantial ionic character. On the other hand, plots of the electron localization function (ELF) (see Supplementary Figure S5) show materialdependent (see below) charge concentration between $\mathrm{As} / \mathrm{Sb}$ and $\mathrm{H}$ atoms. Hence the predicted stable compounds are dominated by mixed covalent and ionic interactions.

3.2. Electronic Structure, Phonons, Electron-Phonon Coupling, and Superconductivity of Identified Stable Pnictogen Hydrides. The DFT calculations indicate that all of the compounds are metallic within their stable pressure ranges. Figure 3 shows band structures and projected densities

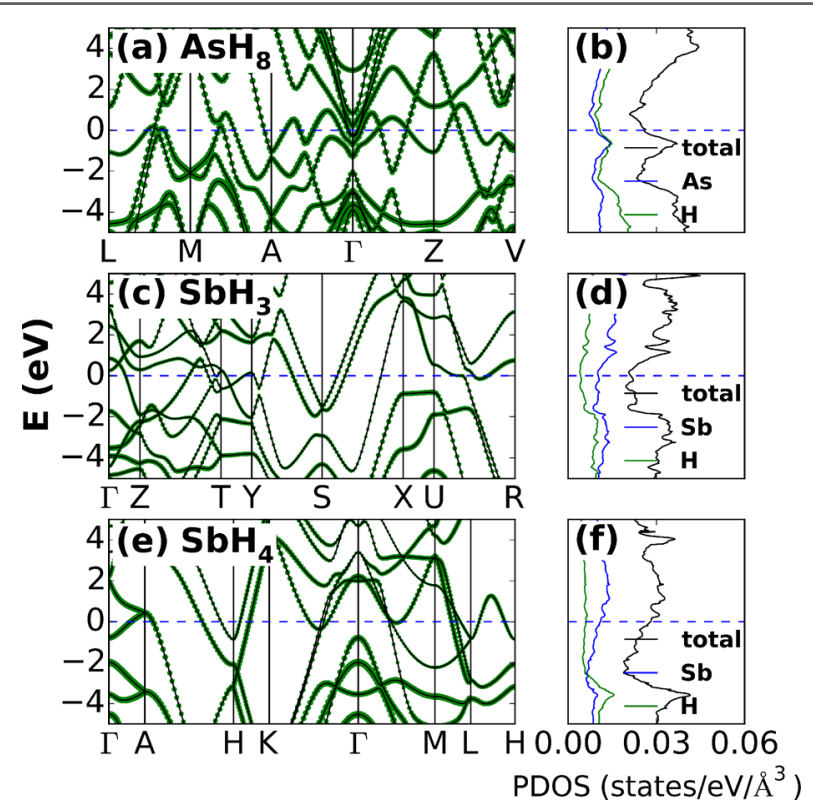

Figure 3. Calculated band structures and projected densities of states of stable $\mathrm{H}$-rich pnictogen hydrides: $(\mathrm{a}, \mathrm{b}) \mathrm{AsH}_{8}$ at $350 \mathrm{GPa},(\mathrm{c}, \mathrm{d})$ $\mathrm{SbH}_{3}$ at $300 \mathrm{GPa}$, and (e,f) $\mathrm{SbH}_{4}$ at $150 \mathrm{GPa}$. The green circles in the band structures represent the orbital projections of the electronic states onto $\mathrm{H}$ atoms.

of states (DOS) for three H-rich compounds, $\mathrm{AsH}_{8}, \mathrm{SbH}_{3}$, and $\mathrm{SbH}_{4}$ (see Supplementary Figure $\mathrm{S} 3$ for results for $\mathrm{H}$-poor AsH and $\mathrm{SbH}$ ). The Fermi levels $\left(\mathrm{E}_{f}\right)$ of $\mathrm{AsH}_{8}$ (Figure 3a-b) and $\mathrm{SbH}_{4}$ (Figure 3e-f) are located at shoulders of a peak in the DOS, leading to a rather large value of the DOS at $\mathrm{E}_{f}\left(\mathrm{~N}\left(\mathrm{E}_{f}\right)\right)$. Moreover, substantial $\mathrm{H}$-derived states exist in proximity to $\mathrm{E}_{f}$ which mimics solid metallic hydrogen. These features are potentially favorable for high-temperature superconductivity. This is, however, not the case for $\mathrm{SbH}_{3}$ (Figure 3c-d) in which $\mathrm{E}_{f}$ lies near the bottom of a broad valley in the DOS, which gives a low $\mathrm{N}\left(\mathrm{E}_{f}\right)$ and only a small amount of $\mathrm{H}$ derived states at $\mathrm{E}_{f}$.

As shown in the phonon spectra of Figure $4\left(\mathrm{a}: \mathrm{AsH}_{8}\right.$, d: $\mathrm{SbH}_{3}$, and $\mathrm{g}: \mathrm{SbH}_{4}$ ) and Supplementary Figure $\mathrm{S} 4$ (AsH and

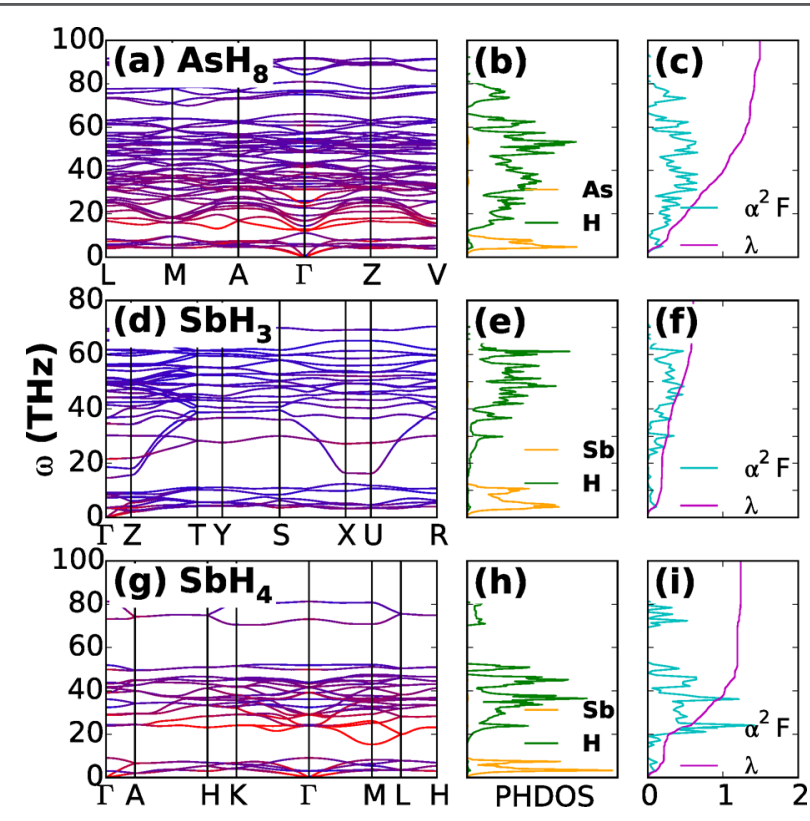

Figure 4. Calculated phonon dispersion curves $(\mathrm{a}, \mathrm{d}, \mathrm{g})$, projected phonon density of states (PHDOS) (b,e,h), Eliashberg EPC spectral functions $\alpha^{2} \mathrm{~F}(\omega)$ and its integral $\lambda(\omega)(\mathrm{c}, \mathrm{f}, \mathrm{i})$ of $\mathrm{AsH}_{8}$ at $350 \mathrm{GPa}$, $\mathrm{SbH}_{3}$ at $300 \mathrm{GPa}$ and $\mathrm{SbH}_{4}$ at $150 \mathrm{GPa}$, respectively. The proportion of red color in the phonon dispersion curves represents the magnitude of the EPC parameter $\lambda_{n, q}(\omega)$ at each phonon mode $(n, q)$.

$\mathrm{SbH})$, all of the compounds are dynamically stable as demonstrated by the absence of imaginary phonon frequencies. The projected phonon DOS of $\mathrm{SbH}_{4}$ (Figure 4h) shows three well separated regions: the low-frequency vibrations associated with heavy $\mathrm{Sb}$ atoms (below $15 \mathrm{THz}$ ), higher frequency wagging, bending, and stretching modes derived from the $\mathrm{H}$ atom bonded to $\mathrm{Sb}$ (between 15 and $65 \mathrm{THz}$ ) and highfrequency intramolecular $\mathrm{H}-\mathrm{H}$ stretching modes from the quasi-molecular $\mathrm{H}_{2}$-units (around $80 \mathrm{THz}$ ). The $\mathrm{H}-\mathrm{H}$ stretching modes of $\mathrm{SbH}_{3}$ (Figure 4e) are lower in frequency, and they merge into the medium-frequency region because of the larger bond lengths of the $\mathrm{H}_{2}$-units. In $\mathrm{AsH}_{8}$ (Figure 4b), two groups of $\mathrm{H}-\mathrm{H}$ stretching modes appear, which correspond to two types of the $\mathrm{H}_{2}$ unit with different bond lengths.

We performed EPC calculations for the predicted stable compounds to probe their superconducting properties. Figure 4 shows the EPC parameters for each phonon mode (a,d,g), the Eliashberg EPC spectral function $\alpha^{2} \mathrm{~F}(\omega)$, and its integral $\lambda(\omega)$ (c,f,i) for $\mathrm{AsH}_{8}, \mathrm{SbH}_{3}$, and $\mathrm{SbH}_{4}$ (results for AsH and $\mathrm{SbH}$ are shown in Supplementary Figure S4). Figure 5a summarizes data for the total EPC parameters $\lambda$ and their pressure dependence. $\mathrm{AsH}_{8}$ and $\mathrm{SbH}_{4}$ are $\mathrm{H}$-rich compounds with rather high $\lambda$ values above 1.0, while $\mathrm{SbH}_{3}$ has a lower value of about 0.5. For all three $\mathrm{H}$-rich compounds the intermediate-frequency $\mathrm{H}$ derived wagging, bending, and stretching phonons and low- 

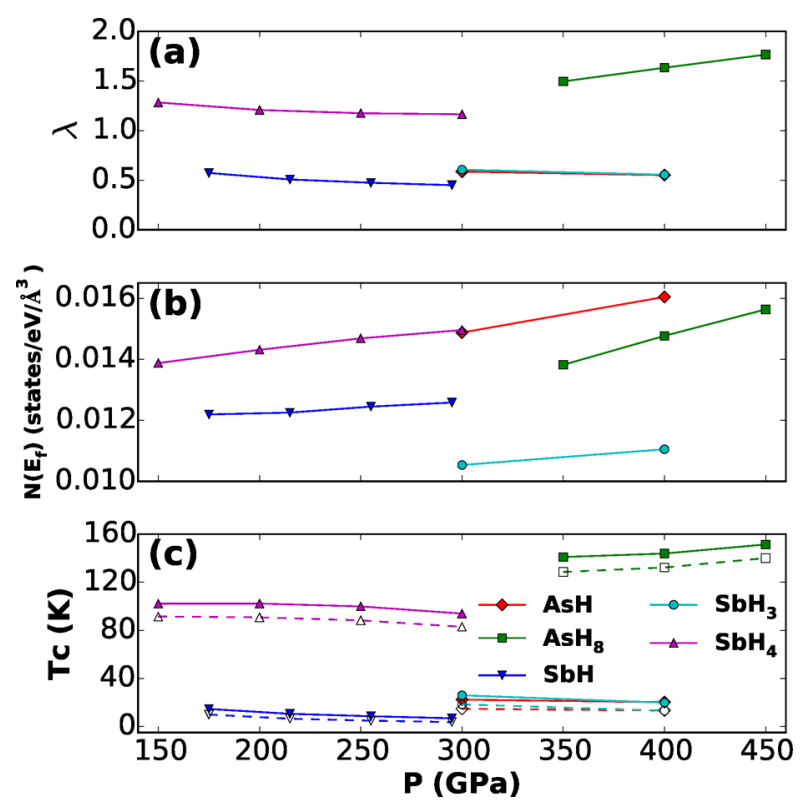

Figure 5. The total EPC parameter $\lambda(\mathrm{a})$, electronic DOS at the Fermi level $\mathrm{N}\left(\mathrm{E}_{f}\right)(\mathrm{b})$, and superconducting critical temperature $T_{c}(\mathrm{c})$ as a function of pressure for the predicted stable compounds. For the $T_{c}$ calculations, two different screened Coulomb repulsion parameters of $\mu^{*}=0.1$ (filled symbols and solid lines) and 0.13 (open symbols and dashed lines) are used for comparison.

frequency vibrations from the heavy $\mathrm{As} / \mathrm{Sb}$ atoms, rather than the high-frequency phonons derived from the $\mathrm{H}_{2}$-units, dominate the contributions to the total EPC.

Within the framework of strong-coupling BCS theory, ${ }^{58}$ the total EPC parameter is proportional to the product of $\mathrm{N}\left(\mathrm{E}_{f}\right)$ and the squared electronic matrix element $\left\langle I^{2}\right\rangle$ averaged over the Fermi surface. The calculated values of $\mathrm{N}\left(\mathrm{E}_{f}\right)$ for each compound are shown in Figure $5 \mathrm{~b}$. While $\mathrm{AsH}_{8}$ and $\mathrm{SbH}_{4}$ have rather high values of $\mathrm{N}\left(\mathrm{E}_{f}\right), \mathrm{SbH}_{3}$ exhibits quite a low $\mathrm{N}\left(\mathrm{E}_{f}\right)$. These results accord with their significantly different $\lambda$ values. The high $\mathrm{N}\left(\mathrm{E}_{f}\right)$ in $\mathrm{H}$-poor AsH arises predominantly from Asinduced states (Supplementary Figure S3b) and results in a small value of $\lambda$ similar to that of superconducting solid As under compression. ${ }^{59,60}$ Besides the difference in $\mathrm{N}\left(\mathrm{E}_{f}\right)$, we find that the strengths of the covalent components of $\mathrm{As} / \mathrm{Sb}-\mathrm{H}$ bonds (with mixed covalent and ionic nature) vary considerably among the compounds, as indicated by the ELF data shown in Supplementary Figure S5. The $\mathrm{Sb}-\mathrm{H}$ bonds in $\mathrm{SbH}_{3}$ are much weaker than those of $\mathrm{SbH}_{4}$ and the $\mathrm{As}-\mathrm{H}$ bonds in $\mathrm{AsH}_{8}$. The weaker $\mathrm{Sb}-\mathrm{H}$ bonds in $\mathrm{SbH}_{3}$ in principle correspond to a low deformation potential, i.e., a smaller modification of the electronic structure, with respect to the motion of $\mathrm{H}$ atoms. This leads to a relatively small $\left\langle I^{2}\right\rangle$ and thus a low $\lambda$.

The superconducting $T_{c}$ values of the stable compounds are evaluated using the Allen-Dynes modified McMillan equation ${ }^{61}$ with calculated logarithmic average frequency $\left(\omega_{\text {log }}\right)$ and a typical choice of screened Coulomb repulsion parameter of $\mu^{*}$ $=0.1$. The results shown in Figure 5c (see Supplementary Table S4 for numerical values) are consistent with the trends in $\lambda$ (Figure 5a). AsH $\mathrm{H}_{8}$ and $\mathrm{SbH}_{4}$ exhibit quite high $T_{c}$ values of around 150 and $100 \mathrm{~K}$, respectively. The other compounds have moderate $T_{c}$ values of $\sim 20 \mathrm{~K}$ or below. The value of $T_{c}$ in $\mathrm{SbH}_{4}$ decreases slightly with pressure, while $\mathrm{AsH}_{8}$ shows an increase. Using a standard value of $\mu^{*}=0.13$ for metallic hydrides, ${ }^{14}$ we find a small decrease in $T_{c}$ with pressure, $\sim 8-$ $11 \%$ in the higher- $T_{c}$ compounds of $\mathrm{AsH}_{8}$ and $\mathrm{SbH}_{4}$.

3.3. Deduced General Chemical Trends of HighPressure Hydrogen-Containing Superconductors. Gathering data for pnictogen hydrides and other hydrides $^{12,22,23,30-38,62,63}$ allows us to deduce useful physical principles about the phase stability and occurrence of hightemperature superconductivity in $\mathrm{H}$-containing materials under pressure. For clarity, here we consider only the binary hydrides $\mathrm{M}_{n} \mathrm{H}_{m}$ formed by main-group elements $\mathrm{M}$ with $s p$ bonding. We find that the Pauling electronegativity difference between $M$ and $\mathrm{H}$ at ambient pressure, $\chi_{M}-\chi_{H}$, is a good "descriptor" of high-pressure phase stability, structural features, and superconducting properties of the hydrides. Specifically, we find that the following properties of $\mathrm{M}_{n} \mathrm{H}_{m}$ are related to $\chi_{M}-\chi_{H}$ :

i. Energetic Stability against Elemental Decomposition. Figure 6 shows formation enthalpies of various hydrides at 200

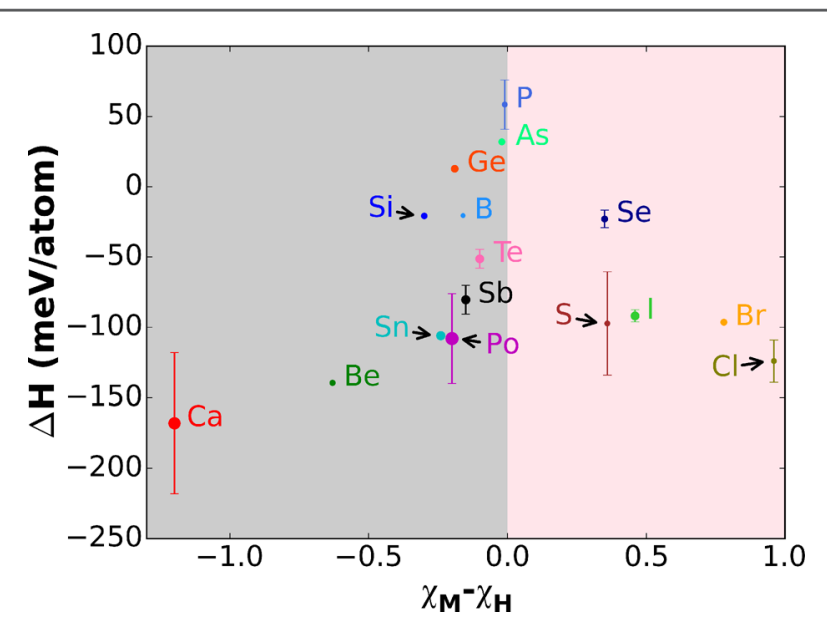

Figure 6. Formation enthalpies $\Delta H$ (in $\mathrm{meV} /$ atom) of main-groupelement (M) binary hydrides ${ }^{12,22,23,30-38,62}$ at $200 \mathrm{GPa}$ with respect to decomposition into the constituent elemental solids, plotted against the electronegativity difference between $\mathrm{M}$ and $\mathrm{H}$ at ambient pressure, $\chi_{M}-\chi_{H}$. The electronegativity values are taken from Pauling's scale. ${ }^{65}$ When several stable phases with different stoichiometries exist, the averaged formation enthalpy is shown, and the end points of the error bars represent the minimum and maximum values of $\Delta H$. The sizes of the circles are proportional to the empirical atomic radii. ${ }^{64}$ The regions corresponding to positive and negative $\chi_{M}-\chi_{H}$ are shown in the background colors of pink and gray, respectively.

GPa as a function of $\chi_{M}-\chi_{H}$. For cases in which more than one stable stoichiometry exists, the averaged formation enthalpy (among all the stoichiometries) is shown, accompanied by an error bar indicating the maximum and minimum enthalpy. The diameter of the circular symbol is proportional to the atomic radius of $\mathrm{M}^{64}$ This divides hydrides into two classes with negative and positive $\chi_{M}-\chi_{H}$, respectively. Considering the extreme cases, $\mathrm{H}_{2} \mathrm{O}$ with $\chi_{M}-\chi_{H} \gg 0$ has strong covalent $\mathrm{O}-\mathrm{H}$ bonds, whereas $\mathrm{LiH}$ with $\chi_{M}-\chi_{\mathrm{H}} \ll 0$ has strong ionic $\mathrm{Li}-\mathrm{H}$ bonding. It may thus be reasonable to conjecture that the hydrides with $\chi_{M}-\chi_{H}>0$ prefer to form covalent $\mathrm{M}-\mathrm{H}$ bonds, while those with $\chi_{M}-\chi_{H}<0$ are more ionic in nature. In the regions of both $\chi_{M}-\chi_{H}>0$ and $\chi_{M}-\chi_{H}<0$, we observe a general trend that the formation enthalpy decreases (i.e., stability increases) with $\left|\chi_{M}-\chi_{H}\right|$. For $\chi_{M}-\chi_{H}>0$, this can be explained by the larger electronegativity of $M$ which corresponds to strongly covalent $\mathrm{M}-\mathrm{H}$ bonds and therefore to higher stability of the hydrides. In this region, the atomic 
radius can also affect the covalent bond strength and therefore have a critical role in determining the stability. For instance, despite the similar electronegativities of $\mathrm{S}$ and $\mathrm{Se}, \mathrm{S}$ hydrides have much lower formation enthalpies than Se hydrides (by about 50-150 meV/atom). We attribute this to the smaller atomic radius of $S$ favoring stronger covalent $\mathrm{H}-\mathrm{S}$ bonds. In the region $\chi_{M}-\chi_{H}<0$, the smaller electronegativity of $\mathrm{M}$ and larger value of $\left|\chi_{M}-\chi_{H}\right|$ leads to substantial charge transfer from the cationic $M$ to anionic $H$. On the one hand, this increases the Madelung energy of the ionic component of the $\mathrm{M}-\mathrm{H}$ bonding, which increases the stability. On the other hand, the charge transfer is favorable for the formation of quasimolecular $\mathrm{H}$ units in the interstitial regions (see below). This lowers the enthalpy of hydrides and make them more stable. This explanation accords with the increased thermodynamic stability of P, As, and Sb hydrides, as demonstrated above.

ii. Key Structural Features. For the hydrides with $\chi_{M}-\chi_{H}<$ 0 , for example our predicted $\mathrm{AsH}_{8}, \mathrm{SbH}_{3}$, and $\mathrm{SbH}_{4}$ phases, there is substantial charge transfer from the relatively electropositive $\mathrm{M}$ to the electronegative $\mathrm{H}$ (Supplementary Table S3). The $\mathrm{M}-\mathrm{H}$ bonds have mixed covalent and ionic nature. With such weaker $\mathrm{M}-\mathrm{H}$ bonding, the $\mathrm{H}$ atoms are prone to move to the interstitial regions under pressures. Therefore, substantial energy gain may be achieved via the formation of $\mathrm{H}-\mathrm{H}$ covalent bonds in the interstitial regions. This is especially the case under pressure where large nonbonded anions are unfavorable. This leads to the formation of intriguing quasi-molecular $\mathrm{H}$-units in $\mathrm{H}$-rich compounds. ${ }^{23,30-33,55,66}$ Turning to $\chi_{M}-\chi_{H}>0$, for instance $\mathrm{S}^{12,16,17}$ and $\mathrm{Se}^{19,22}$ hydrides, the strong polar covalent $\mathrm{M}-\mathrm{H}$ bonds dominate. Under such conditions, all cationic $\mathrm{H}$ atoms are tightly bonded by the $\mathrm{M}$ atoms with large electronegativity, and thus the formation of $\mathrm{H}-\mathrm{H}$ bonds is energetically unfavorable. The quasi-molecular $\mathrm{H}$-unit is absent in the hydrides. The distinction between this key structural behavior in the two regions is reflected in Figure 7, which shows the evolution of the CALYPSO search for $\mathrm{SbH}_{4}\left(\chi_{M}-\chi_{H}<0\right)$ and $\mathrm{SeH}_{3}\left(\chi_{M}-\chi_{H}>0\right)$ at $200 \mathrm{GPa}$. Usually tens of generations are required to determine the global minimum in the CALYPSO calculations. The structures at each generation are sorted according to their enthalpy. The color coding represents the minimum distance between two $\mathrm{H}$ atoms in each structure. All of the low enthalpy structures of $\mathrm{SbH}_{4}$ contain quasi-molecular $\mathrm{H}_{2}$ units with short contacts $(0.8-0.9 \AA)$ between neighboring $\mathrm{H}$ atoms. In contrast, in $\mathrm{SeH}_{3}$ the low-lying structures exhibit large minimum $\mathrm{H}-\mathrm{H}$ distances (over $1.5 \AA$ ) as threedimensional covalent $\mathrm{H}-\mathrm{Se}$ networks are formed.

iii. Density of States at the Fermi Level. As demonstrated in Figure $5 b, N\left(E_{f}\right)$ is a critical parameter in determining the strength of the EPC. Figure 8 shows the values of $\mathrm{N}\left(\mathrm{E}_{f}\right)$ for the binary hydrides that are predicted to be superconductors, $^{12,22,23,30-35,37-39,62,63}$ as a function of $\chi_{M}-\chi_{H}$ at 200 GPa. To make a fair comparison and avoid potential errors arising from different theoretical calculations, we take structural data from refs 12, 22, 23, 30-35, 37-39, 62, and 63 and perform full structural optimizations and calculate $\mathrm{N}\left(\mathrm{E}_{f}\right)$ using the plane-wave PAW method. In general, we find that high values of $\mathrm{N}\left(\mathrm{E}_{f}\right)$ appear in the region with relatively small magnitudes of $\chi_{M}-\chi_{H}$. The compounds with higher values of $\mathrm{N}\left(\mathrm{E}_{f}\right)$ in the two regions are $\mathrm{TeH}_{4}, \mathrm{SbH}_{4}\left(\chi_{M}-\chi_{H}<0\right)$ and $\mathrm{SH}_{3}, \mathrm{SeH}_{3}\left(\chi_{M}-\chi_{H}>0\right)$, respectively. The highly symmetric structures of these hydrides $\left(\mathrm{TeH}_{4}: P 6 / m m m ; \mathrm{SbH}_{4}: \mathrm{Pb}_{3} / m m c\right.$; $\left.\mathrm{S} / \mathrm{SeH}_{3}: \operatorname{Im} \overline{3} m\right)$, which in principle give rise to high degeneracy

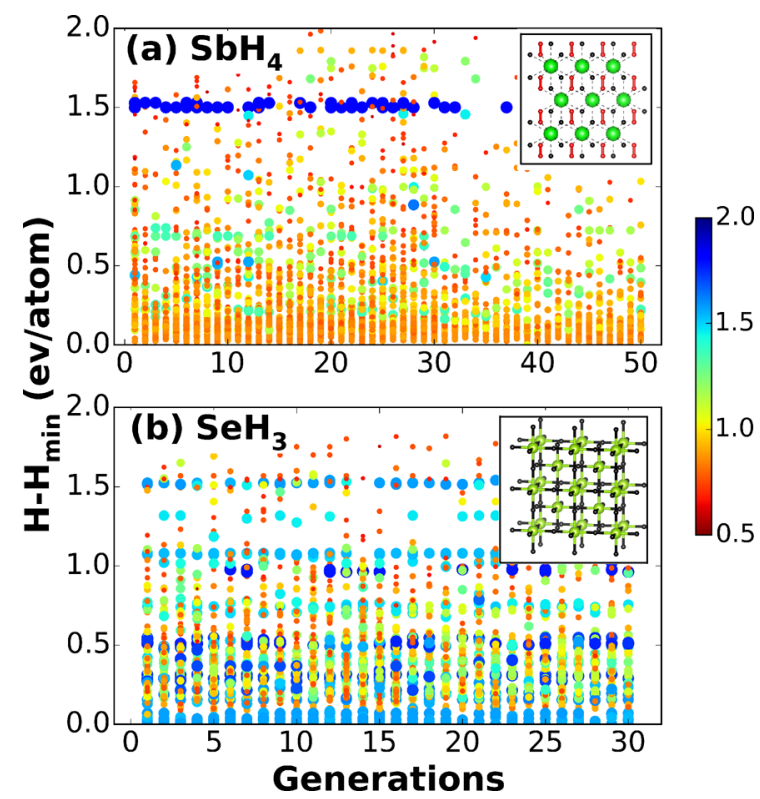

Figure 7. The structures generated are sorted according to enthalpy during the CALYPSO structure searches for $\mathrm{SbH}_{4}(\mathrm{a})$ and $\mathrm{SeH}_{3}(\mathrm{~b})$ at $200 \mathrm{GPa}$, as a function of search generations. The color-coding represents the minimum distance between two $\mathrm{H}$ atoms, which is also proportional to the size of the circle. The insets show the predicted ground-state structures of $\mathrm{SbH}_{4}$ and $\mathrm{SeH}_{3}$.

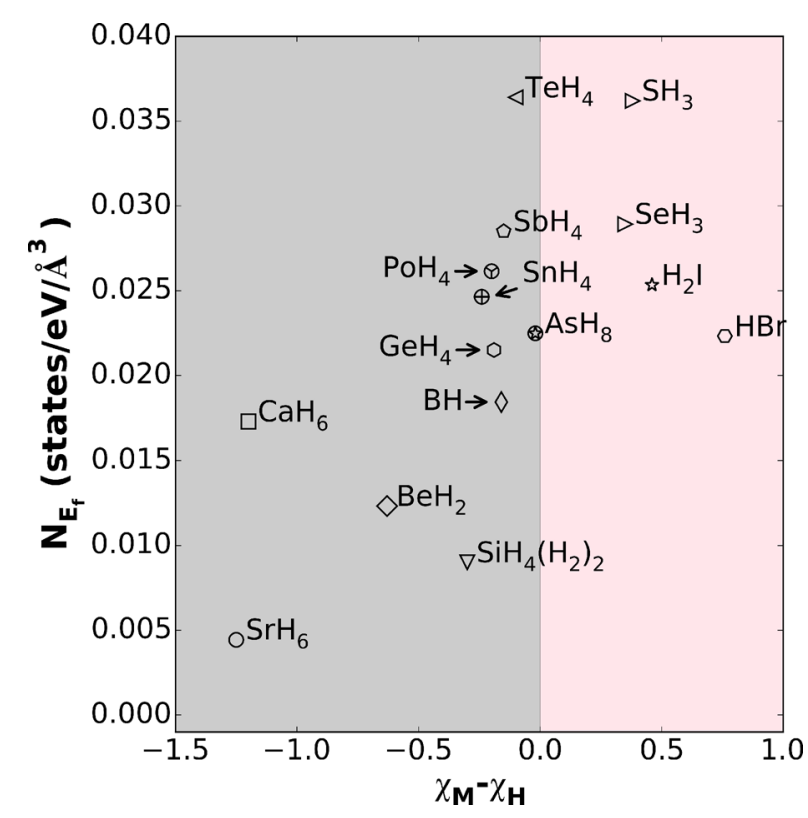

Figure 8. Calculated electronic DOS at the Fermi level $\mathrm{N}\left(\mathrm{E}_{f}\right)$ of maingroup-element binary hydrides ${ }^{12,22,23,30-35,37-39,62,63}$ at $200 \mathrm{GPa}$, as a function of the electronegativity difference between $M$ and $H$ at ambient pressure, $\chi_{M}-\chi_{H}$. When several stable phases with different stoichiometries exist, we choose the one with the highest predicted superconducting $T_{c}$. Similar to Figure 6, the regions of $\chi_{M}-\chi_{H}>0$ and $\chi_{M}-\chi_{H}<0$ are in pink and gray, respectively.

of the band structure at special $k$-points, may be responsible for the high values of $\mathrm{N}\left(\mathrm{E}_{f}\right)$. They are all predicted to be good superconductors with values of $\lambda$ above $1 .{ }^{12,17,19,22,23}$

iv. Features of the Electron-Phonon Coupling. Figure 9 shows the Eliashberg spectral function $\alpha^{2} \mathrm{~F}(\omega)$ (black lines), its normalized integral $\lambda(\omega)$ (divided by the total $\lambda$, colorful scale), 


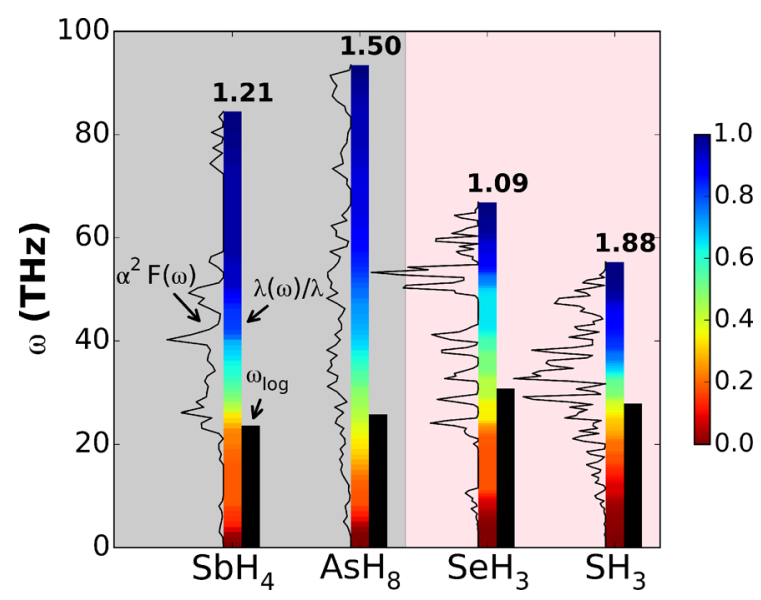

Figure 9. The Eliashberg EPC spectral function $\alpha^{2} \mathrm{~F}(\omega)$ (black lines), its integral $\lambda(\omega)$ (colorful scale), and logarithmic average frequency $\omega_{\text {log }}$ (black scale) for typical hydrides in the regions of $\chi_{M}-\chi_{H}>0$ $\left(\mathrm{SH}_{3}\right.$ and $\left.\mathrm{SeH}_{3}\right)$ and $\chi_{M}-\chi_{\mathrm{H}}<0\left(\mathrm{AsH}_{8}\right.$ and $\left.\mathrm{SbH}_{4}\right)$. For comparison among different compounds, the $\lambda(\omega)$ is normalized and divided by the total $\lambda$ (indicated for each compound).

and logarithmic average frequency $\omega_{\log }$ (black scale) of typical compounds from the two regions. In the $\chi_{M}-\chi_{H}<0$ region $\left(\mathrm{AsH}_{8}\right.$ and $\left.\mathrm{SbH}_{4}\right)$, the total EPC originates mainly from the medium-frequency phonons, predominantly wagging, bending, and stretching modes derived from the $\mathrm{H}$ bonded to As/Sb. The higher frequency $\mathrm{H}-\mathrm{H}$ stretching modes of the quasimolecular $\mathrm{H}_{2}$-units make fairly small contributions to the EPC. Therefore, the integral $\lambda(\omega)$ becomes saturated (in blue) at the upper-middle part of the $\omega$ range. Turning to the region of $\chi_{M}$ $-\chi_{H}>0\left(\mathrm{SH}_{3}\right.$ and $\left.\mathrm{SeH}_{3}\right)$, the EPC arises mainly from the highfrequency $\mathrm{H}$-derived phonons of covalent $\mathrm{M}-\mathrm{H}$ networks. The integral $\lambda(\omega)$ thus becomes saturated close to the upper boundary of $\omega$. These features of the EPC can be ascribed to the different chemical bonding and structural features of the hydrides in the two regions. Note that $\omega_{l o g}$, the important quantity determining the superconducting $T_{c}$, shows higher values in the $\chi_{M}-\chi_{H}>0$ region than in the $\chi_{M}-\chi_{H}<0$ region, despite the evidently lower maximum $\omega$ of the former region than that of the latter. This demonstrates that simply hardening the phonons is not always effective in raising $T_{c}$. The reason originates from the distinct dependence of $\alpha^{2} \mathrm{~F}(\omega)$ on $\omega$ in the two regions, since only the phonons contributing to the EPC are counted when evaluating $T_{c}$.

3.4. More Relevant Discussion. Since Ashcroft first proposed metallic hydrides as potentially good superconductors at high pressures, ${ }^{14}$ high- $T_{c}$ (above $100 \mathrm{~K}$ ) superconductivity has been predicted in a number of high-pressure $\mathrm{H}$-rich compounds. $12,17,22,23,30,31$ The $S$ hydride is one of these predictions $^{16,17}$ that has been confirmed experimentally., ${ }^{5,6}$ In other systems, either low temperature superconductivity ${ }^{41,67}$ or no superconductivity ${ }^{68,69}$ was found. While the underlying reasons for this are not yet settled, one possibility is that the predicted superconducting compounds did not form in highpressure experiments. Confining hydrides or mixtures of hydrogen and other substances in diamond anvil cells up to ultrahigh pressure conditions is technically challenging, and, due to the technical limitations, experimental syntheses may fail even though the theoretically predicted structures are energetically stable. This is highly likely for hydrides with small formation enthalpies, such as those of $\mathrm{Si}, \mathrm{Ge}, \mathrm{B}, \mathrm{Se}$, etc., as indicated in Figure 6.

It is also possible that kinetic processes under pressure inhibit formation of some of the predicted compounds. If so, a highly symmetric structure may be more likely to be synthesized because the energetic barrier for the transition between the symmetric structure and other isomers is usually large. This suggests a strong kinetic stability with respect to variations in the external conditions. From this point of view, $\mathrm{S}$ hydrides are indeed favorable for experimental synthesis since the stable $\mathrm{SH}_{3}$ structure has simultaneously a large formation enthalpy $(\sim 135$ $\mathrm{meV} /$ atom at $200 \mathrm{GPa}$ ) and high symmetry (space group $\operatorname{Im} \overline{3} m)$. In this sense, the $\mathrm{SbH}_{4}$ phase predicted in the present work is quite promising for possible experimental synthesis, in view of its large formation enthalpy $(\sim 90 \mathrm{meV} /$ atom at 200 $\mathrm{GPa}$ ) and highly symmetric structure (space group $\mathrm{P6}_{3} / \mathrm{mmc}$ ). Certainly the larger barrier associated with the highly symmetric structure may make its synthesis challenging. This can be basically overcome by for instance sufficient thermal energy or the assistance of a catalyst.

Turning to trends, results from the current and previous theoretical studies reveal a connection between the thermodynamic stability, structural features, and superconducting properties of high-pressure hydrides and the Pauling electronegativity differences between $\mathrm{H}$ and the other elements. This is a remarkable and at first sight a strange finding, since highpressure chemistry is well-known to be radically different from that at ambient pressure. ${ }^{70}$ Our findings indicate that the ambient-pressure chemical properties determine to a large extent the high-pressure behavior.

Electropositive elements (with $\chi_{M}-\chi_{H} \ll 0$ in our classification), such as alkaline and alkaline earth metals, form highly ionic ambient-pressure hydrides characterized by large charge transfers and structures similar to fluorides. In particular, the crystals are stabilized by the Madelung energy of the cationic $\mathrm{M}$ and anionic $\mathrm{H}$ ions. The anionic $\mathrm{H}$ in these materials are well separated from other $\mathrm{H}$ ions, and the $\mathrm{H}$ vibrational frequencies are relatively low. At high pressures the distances between $\mathrm{H}$ anions are significantly shortened, but the weak ionic $\mathrm{M}-\mathrm{H}$ bonds allow $\mathrm{H}$ atoms to move to the interstitial regions. These lead to stabilization of the $\mathrm{H}-\mathrm{H}$ covalent bonds in the interstitial region. Thus, quasi-molecular aggregation of $\mathrm{H}$ emerges, ${ }^{30,55,63,66}$ and high $\mathrm{H}$ vibrational frequencies occur in the high-pressure phases.

Nonmetallic strongly electronegative elements (with $\chi_{M}-\chi_{H}$ $\gg 0$ ) often form weakly bound insulating $\mathrm{H}$-containing molecular solids at ambient pressure, e.g., $\mathrm{NH}_{3}, \mathrm{H}_{2} \mathrm{O}, \mathrm{H}_{2} \mathrm{~S}$, etc. While strong covalent $\mathrm{M}-\mathrm{H}$ bonds dominate molecules, the intermolecular bonds characterizing cohesion of molecular solids are weak van der Waals bonds and hydrogen bonds. Under compression, with decreasing intermolecular distances and accompanying hydrogen-bond symmetrization, ${ }^{71-73}$ solids with covalent $\mathrm{M}-\mathrm{H}$ bonding networks eventually become energetically favored. Because of the strong polar covalent $\mathrm{M}-$ $\mathrm{H}$ bonds, the $\mathrm{H}-\mathrm{H}$ bonds and aggregation of $\mathrm{H}$ atoms are energetically unfavorable.

As for the elements with moderate electronegativities comparable to that of $\mathrm{H}\left(\chi_{M}-\chi_{H} \sim 0\right)$, the hydrides formed are intermediate between the above two cases. The $\mathrm{M}-\mathrm{H}$ bonds have mixed covalent and ionic characters, and the former is not strong enough to stabilize covalently bonded $\mathrm{M}-\mathrm{H}$ networks. In the hydrides with $\chi_{M}-\chi_{H}<0$ (Si, Ge, Sn, Te, Sb, etc.), the anionic nature of $\mathrm{H}$ atoms (due to the charge transfer 
from $\mathrm{M}$ to $\mathrm{H}$ ) and the tendency of $\mathrm{H}$ atoms to move to interstitial regions (due to the relatively weak $\mathrm{M}-\mathrm{H}$ bonds) under pressure allows aggregation of $\mathrm{H}$ via formation of $\mathrm{H}-\mathrm{H}$ covalent bonds in H-rich phases. This mechanism helps to stabilize hydrides. The stability increases with the magnitude of $\chi_{M}-\chi_{H}$ (as in Figure 6), which determines the Madelung energy of the ionic interaction and the capability of hydrides to form aggregates of $\mathrm{H}$. For As and $\mathrm{P}$ hydrides with small $\chi_{M}-$ $\chi_{\mathrm{H}} \mathrm{l}$, only limited charge transfer from As/P to $\mathrm{H}$ can occur due to the small electronegativity difference. On the other hand, the fairly strong As/ $\mathrm{P}-\mathrm{H}$ covalent bonds hinder $\mathrm{H}$ atoms from moving to interstitial regions. Hence $\mathrm{H}-\mathrm{H}$ covalent bonds are unlikely to form. This may be responsible for their positive formation enthalpies in Figure 6.

Once metallic hydrides are stabilized by high pressure, one can ask whether or not they are good superconductors. Our current results indicate that three features of hydrides are essential for strong EPC and a high $T_{c}$. The first is a high $\mathrm{N}\left(\mathrm{E}_{f}\right)$. While this quantity certainly depends on the specific band structure and electronic occupation, highly symmetric structures are in principle favorable for high values of $\mathrm{N}\left(\mathrm{E}_{f}\right)$. Interestingly this occurs with small magnitudes of $\left|\chi_{M}-\chi_{H}\right|$ (Figure 8). The second feature is the appearance of substantial $\mathrm{H}$-derived states in proximity to $\mathrm{E}_{f}$, mimicking pure metallic hydrogen. H-rich compounds are more likely to satisfy this criterion. The third feature is the large electronic deformation potential with respect to motion of $\mathrm{H}$ atoms. The hydrides with strong chemical bonding, especially covalent bonding, are more favored by this feature. In this regard, the $T_{c}$ of $S$ hydrides are predicted to be enhanced by alloying with more electronegative elements (e.g., $\mathrm{P}$ and $\mathrm{O})^{11,74}$ to further strengthen the covalent bonding. For the hydrides containing quasi-molecular assemblies of $\mathrm{H}$ atoms, ${ }^{23,30-34,55,63,66,75}$ the deformation potential associated with intramolecular $\mathrm{H}-\mathrm{H}$ stretching motion is fairly large (due to the strong $\mathrm{H}-\mathrm{H}$ covalent bonds). Unfortunately, as demonstrated in Figure 9, the corresponding phonon modes make a quite limited contribution to the EPC. An even higher superconducting $T_{c}$ would be expected if these phonon modes were involved in the EPC, for example, under further compression.

While preparing this manuscript, our attention was drawn to two reports on superconductivity in pnictogen hydrides. One is a preliminary observation of high $T_{c}$ superconductivity in $\mathrm{P}$ hydrides from $\sim 30 \mathrm{~K}$ increasing continuously to more than 100 $\mathrm{K}$ up to pressures above $200 \mathrm{GPa} .{ }^{76}$ While more experimental data are required to verify this finding, the basic idea coincides with the main motivation of this work. However, based on our structure searching results, $\mathrm{P}$ hydrides are predicted to be unstable against elemental decomposition up to $400 \mathrm{GPa}$. Our results point to the possibility that $\mathrm{P}$ hydrides formed in the experiments ${ }^{76}$ may be metastable and could be stabilized by kinetic processes at high pressures. The conclusion that the $\mathrm{P}$ hydrides are thermodynamically unstable at megabar pressures is consistent with recent theoretical reports. ${ }^{77,78}$ Further analysis of the metastability and energy barriers related to kinetic stability, as well as superconductivity calculations for $\mathrm{P}$ hydrides, will be published elsewhere. Another is the theoretical prediction of $106 \mathrm{~K}$ superconductivity in $\mathrm{SbH}_{4}$ at $150 \mathrm{GPa}$ by Ma et al. ${ }^{79}$ Using a structure searching method based on a genetic algorithm, ${ }^{80}$ they obtained the same high-symmetry structure (space group $\mathrm{PG}_{3} / \mathrm{mmc}$ ) of $\mathrm{SbH}_{4}$ that we have found in our work. Their calculations of superconducting properties also agree with ours. Experimental attempts to synthesize $\mathrm{Sb}$ hydrides at high pressures and explore their superconducting properties are eagerly anticipated to test this theoretical prediction.

\section{CONCLUSIONS}

We have used first-principles structure searching methods to study the hitherto unknown phase diagram of solid pnictogens (i.e., P, As, Sb) hydrides at high pressures with the aim of predicting new high- $T_{c}$ superconductors. Surprisingly, except for the molecular solid phases stabilized by weak van der Waals interactions near ambient pressure, we did not find any $\mathrm{P}$ hydrides to be stable with respect to decomposition into the elements up to $400 \mathrm{GPa}$. The hydrides containing the heavy element $\mathrm{Sb}$ are found to be the most stable at high pressures. This was unexpected because at ambient pressure the lighter pnictogen hydrides are the most stable.

We have predicted several stable superconducting hydrides (AsH, $\mathrm{AsH}_{8}, \mathrm{SbH}, \mathrm{SbH}_{3}$, and $\mathrm{SbH}_{4}$ ). Among them, two H-rich compounds, $\mathrm{AsH}_{8}$ and $\mathrm{SbH}_{4}$, are predicted to exhibit $T_{c}$ values of $\sim 150 \mathrm{~K}$ above $350 \mathrm{GPa}$ and $\sim 100 \mathrm{~K}$ above $150 \mathrm{GPa}$, respectively. $\mathrm{SbH}_{4}$ is energetically very stable and adopts a highly symmetrical hexagonal structure, and the stabilization pressure is well within reach of modern diamond anvil cell experiments. Finally, through systematic analysis of current and previous studies of binary hydrides, we determined a close connection between the high-pressure behavior of hydrides and ambient pressure chemical quantities. In particular we found that the electronegativity difference between the constituent elements is a good descriptor for characterizing the structure, chemical bonding, thermodynamic stability, and superconducting properties of hydrides at high pressures. Analyzing these trends could provide further insights into the intriguing and diverse chemical and physical properties of compressed hydrides. Our work provides a useful roadmap for discovering more stable compressed hydrides and exploration of their superconducting properties.

\section{ASSOCIATED CONTENT}

\section{Supporting Information}

The Supporting Information is available free of charge on the ACS Publications website at DOI: 10.1021/acs.chemmater.5b04638.

Effect of zero point energy on phase stabilities, electronic structure, phonon, electron-phonon coupling results of $\mathrm{H}$-poor compounds, electron localization function and Bader charge analysis results, explicit structural information and calculated superconducting properties of the identified stable hydrides (PDF)

\section{AUTHOR INFORMATION}

\section{Corresponding Authors}

*E-mail: lijun_zhang@jlu.edu.cn (L.Z.).

*E-mail: singhdj@@missouri.edu (D.J.S.).

*E-mail: mym@jlu.edu.cn (Y.M.).

\section{Author Contributions}

Y.F. and X.D. contributed equally to this work.

\section{Notes}

The authors declare no competing financial interest. 


\section{ACKNOWLEDGMENTS}

The authors thank Eva Zurek for sharing structure data for iodine hydride. The work at Jilin University is supported by the funding of National Natural Science Foundation of China under Grant Nos. 11274136 and 11534003, 2012 Changjiang Scholar of Ministry of Education and the Postdoctoral Science Foundation of China under grant 2013M541283. L.Z. acknowledges funding support from the Recruitment Program of Global Youth Experts in China. Part of the calculations was performed in the high performance computing center of Jilin University. R.J.N. acknowledges financial support from the Engineering and Physical Sciences Research Council (EPSRC) of the UK [EP/J017639/1]. R.J.N. and C.J.P. acknowledge use of the Archer facility of the U.K.'s national high-performance computing service (for which access was obtained via the UKCP consortium [EP/K014560/1]).

\section{REFERENCES}

(1) Kamihara, Y.; Watanabe, T.; Hirano, M.; Hosono, H. Iron-Based Layered Superconductor $\mathrm{La}\left[\mathrm{O}_{1-x} \mathrm{~F}_{x}\right] \mathrm{FeAs}(\mathrm{x}=0.05-0.12)$ with $\mathrm{T}_{c}=$ 26 K. J. Am. Chem. Soc. 2008, 130, 3296-3297.

(2) Fang, C.; Yao, H.; Tsai, W.-F.; Hu, J.; Kivelson, S. A. Theory of electron nematic order in LaFeAsO. Phys. Rev. B: Condens. Matter Mater. Phys. 2008, 77, 224509.

(3) Mazin, I. I.; Singh, D. J.; Johannes, M. D.; Du, M. H. Unconventional Superconductivity with a Sign Reversal in the Order Parameter of LaFeAsO ${ }_{1-x} \mathrm{~F}_{x}$. Phys. Rev. Lett. 2008, 101, 057003.

(4) Scalapino, D. J. A common thread: The pairing interaction for unconventional superconductors. Rev. Mod. Phys. 2012, 84, 13831417.

(5) Drozdov, A. P.; Eremets, M. I.; Troyan, I. A.; Ksenofontov, V.; Shylin, S. I. Conventional superconductivity at $203 \mathrm{~K}$ at high pressures in the sulfur hydride system. Nature 2015, 525, 73.

(6) Einaga, M.; Sakata, M.; Ishikawa, T.; Shimizu, K.; Eremets, M.; Drozdov, A.; Troyan, I.; Hirao, N.; Ohishi, Y. Crystal Structure of 200 K-Superconducting Phase of Sulfur Hydride System. 2015, arXiv: 1509.03156

(7) Nicol, E. J.; Carbotte, J. P. Comparison of pressurized sulfur hydride with conventional superconductors. Phys. Rev. B: Condens. Matter Mater. Phys. 2015, 91, 220507.

(8) Bianconi, A.; Jarlborg, T. Lifshitz transitions and zero point lattice fluctuations in sulfur hydride showing near room temperature superconductivity. 2015, arXiv:1507.01093.

(9) Hirsch, J.; Marsiglio, F. Hole superconductivity in and other sulfides under high pressure. Phys. C 2015, 511, 45-49.

(10) Bernstein, N.; Hellberg, C. S.; Johannes, M. D.; Mazin, I. I.; Mehl, M. J. What superconducts in sulfur hydrides under pressure and why. Phys. Rev. B: Condens. Matter Mater. Phys. 2015, 91, 060511.

(11) Heil, C.; Boeri, L. Influence of bonding on superconductivity in high-pressure hydrides. Phys. Rev. B: Condens. Matter Mater. Phys. 2015, 92, 060508.

(12) Errea, I.; Calandra, M.; Pickard, C. J.; Nelson, J.; Needs, R. J.; Li, Y.; Liu, H.; Zhang, Y.; Ma, Y.; Mauri, F. High-Pressure Hydrogen Sulfide from First Principles: A Strongly Anharmonic PhononMediated Superconductor. Phys. Rev. Lett. 2015, 114, 157004.

(13) Ashcroft, N. W. Metallic Hydrogen: A High-Temperature Superconductor? Phys. Rev. Lett. 1968, 21, 1748-1749.

(14) Ashcroft, N. W. Hydrogen Dominant Metallic Alloys: High Temperature Superconductors? Phys. Rev. Lett. 2004, 92, 187002.

(15) Feng, J.; Grochala, W.; Jaroń, T.; Hoffmann, R.; Bergara, A.; Ashcroft, N. W. Structures and Potential Superconductivity in $\mathrm{SiH}_{4}$ at High Pressure: En Route to "Metallic Hydrogen. Phys. Rev. Lett. 2006, 96, 017006.

(16) Li, Y.; Hao, J.; Liu, H.; Li, Y.; Ma, Y. The metallization and superconductivity of dense hydrogen sulfide. J. Chem. Phys. 2014, 140, 174712 .
(17) Duan, D.; Liu, Y.; Tian, F.; Li, D.; Huang, X.; Zhao, Z.; Yu, H.; Liu, B.; Tian, W.; Cui, T. Pressure-induced metallization of dense $\left(\mathrm{H}_{2} \mathrm{~S}\right)_{2} \mathrm{H}_{2}$ with high- $\mathrm{T}_{c}$ superconductivity. Sci. Rep. 2014, 4, 6968.

(18) Papaconstantopoulos, D. A.; Klein, B. M.; Mehl, M. J.; Pickett, W. E. Cubic $\mathrm{H}_{3} \mathrm{~S}$ around $200 \mathrm{GPa}$ : An atomic hydrogen superconductor stabilized by sulfur. Phys. Rev. B: Condens. Matter Mater. Phys. 2015, 91, 184511.

(19) Flores-Livas, J. A.; Sanna, A.; Gross, E. K. U. High temperature superconductivity in sulfur and selenium hydrides at high pressure. 2015, arXiv:1501.06336.

(20) Wang, Y.; Ma, Y. Perspective: Crystal structure prediction at high pressures. J. Chem. Phys. 2014, 140, 040901.

(21) Duan, D.; Huang, X.; Tian, F.; Li, D.; Yu, H.; Liu, Y.; Ma, Y.; Liu, B.; Cui, T. Pressure-induced decomposition of solid hydrogen sulfide. Phys. Rev. B: Condens. Matter Mater. Phys. 2015, 91, 180502.

(22) Zhang, S.; Wang, Y.; Zhang, J.; Liu, H.; Zhong, X.; Song, H.-F.; Yang, G.; Zhang, L.; Ma, Y. Phase Diagram and High-Temperature Superconductivity of Compressed Selenium Hydrides. 2015, arXiv: 1502.02607

(23) Zhong, X.; Wang, H.; Zhang, J.; Liu, H.; Zhang, S.; Song, H.-F.; Yang, G.; Zhang, L.; Ma, Y. Tellurium Hydrides at High Pressures: High-temperature Superconductors. 2015, arXiv:1503.00396.

(24) Natta, G.; Casazza, E. La struttura dell'idrogeno fosforato $\left(\mathrm{PH}_{3}\right)$ e dell'idrogeno arsenicale $\left(\mathrm{AsH}_{3}\right)$. Gazz. Chim. Ital. 1930, 60, 981859.

(25) Sennikov, P. Weak hydrogen-bonding by second-row $\left(\mathrm{PH}_{3}\right.$, $\left.\mathrm{H}_{2} \mathrm{~S}\right)$ and third-row $\left(\mathrm{AsH}_{3}, \mathrm{H}_{2} \mathrm{Se}\right)$ hydrides. J. Phys. Chem. 1994, 98, 4973-4981.

(26) Baudler, M.; Glinka, K. Contributions to the chemistry of phosphorus. 218. Monocyclic and polycyclic phosphines. Chem. Rev. 1993, 93, 1623-1667.

(27) Holleman, A.; Wiberg, E. Inorganic Chemistry; Academic Press: München, 2001.

(28) Matthias, B. Progress in low temperature physics; North Holland Publishing Company: Leiden, 1957; Vol. 2.

(29) Pines, D. Superconductivity in the Periodic System. Phys. Rev. 1958, 109, 280-287.

(30) Wang, H.; Tse, J. S.; Tanaka, K.; Iitaka, T.; Ma, Y. Superconductive sodalite-like clathrate calcium hydride at high pressures. Proc. Natl. Acad. Sci. U. S. A. 2012, 109, 6463-6466.

(31) Li, Y.; Gao, G.; Xie, Y.; Ma, Y.; Cui, T.; Zou, G. Superconductivity at similar to $100 \mathrm{~K}$ in dense $\mathrm{SiH}_{4}\left(\mathrm{H}_{2}\right)_{2}$ predicted by first principles. Proc. Natl. Acad. Sci. U. S. A. 2010, 107, 1570815711.

(32) Gao, G.; Oganov, A. R.; Bergara, A.; Martinez-Canales, M.; Cui, T.; Iitaka, T.; Ma, Y.; Zou, G. Superconducting High Pressure Phase of Germane. Phys. Rev. Lett. 2008, 101, 107002.

(33) Gao, G.; Oganov, A. R.; Li, P.; Li, Z.; Wang, H.; Cui, T.; Ma, Y.; Bergara, A.; Lyakhov, A. O.; Iitaka, T.; Zou, G. High-pressure crystal structures and superconductivity of Stannane $\left(\mathrm{SnH}_{4}\right)$. Proc. Natl. Acad. Sci. U. S. A. 2010, 107, 1317-1320.

(34) Liu, Y.; Duan, D.; Tian, F.; Li, D.; Sha, X.; Zhao, Z.; Zhang, H.; Wu, G.; Yu, H.; Liu, B.; Cui, T. Phase diagram and superconductivity of polonium hydrides under high pressure. 2015, arXiv:1503.08587.

(35) Shamp, A.; Zurek, E. Superconducting High Pressure Phases Composed of Hydrogen and Iodine. 2015, arXiv:1507.02616.

(36) Duan, D.; Tian, F.; Huang, X.; Li, D.; Yu, H.; Liu, Y.; Ma, Y.; Liu, B.; Cui, T. Decomposition of solid hydrogen bromide at high pressure. 2015, arXiv:1504.01196.

(37) Wang, Z.; Yao, Y.; Zhu, L.; Liu, H.; Iitaka, T.; Wang, H.; Ma, Y. Metallization and superconductivity of $\mathrm{BeH}_{2}$ under high pressure. $J$. Chem. Phys. 2014, 140, 124707.

(38) Hu, C.-H.; Oganov, A. R.; Zhu, Q.; Qian, G.-R.; Frapper, G.; Lyakhov, A. O.; Zhou, H.-Y. Pressure-Induced Stabilization and Insulator-Superconductor Transition of BH. Phys. Rev. Lett. 2013, 110, 165504.

(39) Lu, S.; Wu, M.; Liu, H.; Tse, J. S.; Yang, B. Prediction of novel crystal structures and superconductivity of compressed HBr. RSC Adv. 2015, 5, 45812-45816. 
(40) Gao, L.; Xue, Y. Y.; Chen, F.; Xiong, Q.; Meng, R. L.; Ramirez, D.; Chu, C. W.; Eggert, J. H.; Mao, H. K. Superconductivity up to 164 $\mathrm{K}$ in $\mathrm{HgBa}_{2} \mathrm{Ca}_{m}-1 \mathrm{Cu}_{m} \mathrm{O}_{2 m+2+\delta}(\mathrm{m}=1,2$, and 3$)$ under quasihydrostatic pressures. Phys. Rev. B: Condens. Matter Mater. Phys. 1994, 50, 4260-4263.

(41) Eremets, M. I.; Trojan, I. A.; Medvedev, S. A.; Tse, J. S.; Yao, Y. Superconductivity in Hydrogen Dominant Materials: Silane. Science 2008, 319, 1506-1509.

(42) Degtyareva, O.; Proctor, J. E.; Guillaume, C. L.; Gregoryanz, E.; Hanfland, M. Formation of transition metal hydrides at high pressures. Solid State Commun. 2009, 149, 1583-1586.

(43) Szczesniak, D.; Zemia, T. P. On the high-pressure superconducting phase in platinum hydride. Supercond. Sci. Technol. 2015, 28, 085018 .

(44) Wang, Y.; Lv, J.; Zhu, L.; Ma, Y. Crystal structure prediction via particle-swarm optimization. Phys. Rev. B: Condens. Matter Mater. Phys. 2010, 82, 094116.

(45) Wang, Y.; Lv, J.; Zhu, L.; Ma, Y. CALYPSO: A method for crystal struc- ture prediction. Comput. Phys. Commun. 2012, 183, 2063-2070.

(46) Pickard, C. J.; Needs, R. J. High-Pressure Phases of Silane. Phys. Rev. Lett. 2006, 97, 045504.

(47) Pickard, C. J.; Needs, R. J. Ab initio random structure searching. J. Phys.: Condens. Matter 2011, 23, 053201.

(48) Kresse, G.; Furthmüller, J. Efficient iterative schemes for $a b$ initio total-energy calculations using a plane-wave basis set. Phys. Rev. B: Condens. Matter Mater. Phys. 1996, 54, 11169-11186.

(49) Blöchl, P. E. Projector augmented-wave method. Phys. Rev. B: Condens. Matter Mater. Phys. 1994, 50, 17953-17979.

(50) Perdew, J. P.; Chevary, J. A.; Vosko, S. H.; Jackson, K. A.; Pederson, M. R.; Singh, D. J.; Fiolhais, C. Atoms, molecules, solids, and surfaces: Applications of the generalized gradient approximation for exchange and correlation. Phys. Rev. B: Condens. Matter Mater. Phys. 1992, 46, 6671-6687.

(51) Giannozzi, P.; Baroni, S.; Bonini, N.; Calandra, M.; Car, R.; Cavazzoni, C.; Ceresoli, D.; Chiarotti, G. L.; Cococcioni, M.; Dabo, I.; Corso, A. D.; de Gironcoli, S.; Fabris, S.; Fratesi, G.; Gebauer, R.; Gerstmann, U.; Gougoussis, C.; Kokalj, A.; Lazzeri, M.; Martin-Samos, L.; Marzari, N.; Mauri, F.; Mazzarello, R.; Paolini, S.; Pasquarello, A.; Paulatto, L.; Sbraccia, C.; Scandolo, S.; Sclauzero, G.; Seitsonen, A. P.; Smogunov, A.; Umari, P.; Wentzcovitch, R. M. QUANTUM ESPRESSO: a modular and open-source software project for quantum simulations of materials. J. Phys.: Condens. Matter 2009, 21, 395502.

(52) Methfessel, M.; Paxton, A. T. High-precision sampling for Brillouin-zone integration in metals. Phys. Rev. B: Condens. Matter Mater. Phys. 1989, 40, 3616-3621.

(53) Katzke, H.; Tolédano, P. Displacive mechanisms and orderparameter symmetries for the A7-incommensurate-bcc sequences of high-pressure reconstructive phase transitions in Group Va elements. Phys. Rev. B: Condens. Matter Mater. Phys. 2008, 77, 024109.

(54) Pickard, C. J.; Needs, R. J. Structure of phase III of solid hydrogen. Nat. Phys. 2007, 3, 473-476.

(55) Zurek, E.; Hoffmann, R.; Ashcroft, N. W.; Oganov, A. R.; Lyakhov, A. O. A little bit of lithium does a lot for hydrogen. Proc. Natl. Acad. Sci. U. S. A. 2009, 106, 17640-17643.

(56) Tse, J. S.; Yao, Y.; Tanaka, K. Novel Superconductivity in Metallic $\mathrm{SnH}_{4}$ under High Pressure. Phys. Rev. Lett. 2007, 98, 117004.

(57) Tang, W.; Sanville, E.; Henkelman, G. A grid-based Bader analysis algorithm without lattice bias. J. Phys.: Condens. Matter 2009, 21,084204 .

(58) McMillan, W. L. Transition Temperature of Strong-Coupled Superconductors. Phys. Rev. 1968, 167, 331-344.

(59) Chen, A. L.; Lewis, S. P.; Su, Z.; Yu, P. Y.; Cohen, M. L. Superconductivity in arsenic at high pressures. Phys. Rev. B: Condens. Matter Mater. Phys. 1992, 46, 5523-5527.

(60) Berman, I. V.; Brandt, N. B. Superconductivity of Arsenic at High Pressures. J. Exp. Theor. Phys. Lett. 1969, 10, 55.

(61) Allen, P. B.; Dynes, R. C. Transition temperature of strongcoupled superconductors reanalyzed. Phys. Rev. B 1975, 12, 905-922.
(62) Wang, Z.; Wang, H.; Tse, J. S.; Iitaka, T.; Ma, Y. Stabilization of $\mathrm{H}_{3}^{+}$in the high pressure crystalline structure of $\mathrm{H}_{n} \mathrm{Cl}(\mathrm{n}=2-7)$. Chem. Sci. 2015, 6, 522-526.

(63) Wang, Y.; Wang, H.; Tse, J. S.; Iitaka, T.; Ma, Y. Structural morphologies of high-pressure polymorphs of strontium hydrides. Phys. Chem. Chem. Phys. 2015, 17, 19379-19385.

(64) Slater, J. C. Atomic Radii in Crystals. J. Chem. Phys. 1964, 41, 3199-3204.

(65) Lide, D. R. CRC handbook of chemistry and physics; CRC Press: Boca Raton, 2004.

(66) Baettig, P.; Zurek, E. Pressure-Stabilized Sodium Polyhydrides: $\mathrm{NaH}_{n}(n>1)$. Phys. Rev. Lett. 2011, 106, 237002.

(67) Muramatsu, T.; Wanene, W. K.; Somayazulu, M.; Vinitsky, E.; Chandra, D.; Strobel, T. A.; Struzhkin, V. V.; Hemley, R. J. Metallization and Superconductivity in the Hydrogen-Rich Ionic Salt $\mathrm{BaReH}_{9}$. J. Phys. Chem. C 2015, 119, 18007-18013.

(68) Goncharenko, I.; Eremets, M. I.; Hanfland, M.; Tse, J. S.; Amboage, M.; Yao, Y.; Trojan, I. A. Pressure-Induced HydrogenDominant Metallic State in Aluminum Hydride. Phys. Rev. Lett. 2008, $100,045504$.

(69) Eremets, M. I.; Struzhkin, V. V.; kwang Mao, H.; Hemley, R. J. Exploring superconductivity in low- $\mathrm{Z}$ materials at megabar pressures. Phys. B 2003, 329-333, 1312-1316.

(70) Van Eldik, R.; Klärner, F.-G. High pressure chemistry; John Wiley \& Sons: Erlangen, 2008.

(71) Lee, C.; Vanderbilt, D.; Laasonen, K.; Car, R.; Parrinello, M. Ab initio studies on high pressure phases of ice. Phys. Rev. Lett. 1992, 69, $462-465$.

(72) Aoki, K.; Yamawaki, H.; Sakashita, M.; Fujihisa, H. Infrared absorption study of the hydrogen-bond symmetrization in ice to 110 GPa. Phys. Rev. B: Condens. Matter Mater. Phys. 1996, 54, 1567315677.

(73) Hirsch, K. R.; Holzapfel, W. B. Effect of high pressure on the Raman spectra of ice VIII and evidence for ice X. J. Chem. Phys. 1986, 84, 2771-2775.

(74) Ge, Y.; Zhang, F.; Yao, Y. Possible Superconductivity Approaching Ice Point. 2015, arXiv:1507.08525.

(75) Struzhkin, V. V.; Kim, D.; Stavrou, E.; Muramatsu, T.; Mao, H.; Pickard, C. J.; Needs, R. J.; Prakapenka, V. B.; Goncharov, A. F. Synthesis of Sodium Polyhydrides at High Pressures. 2014, arXiv: 1412.1542 .

(76) Drozdov, A. P.; Eremets, M. I.; Troyan, I. A. Superconductivity above $100 \mathrm{~K}$ in $\mathrm{PH}_{3}$ at high pressures. 2015, arXiv:1508.06224.

(77) Shamp, A.; Terpstra, T.; Bi, T.; Falls, Z.; Avery, P.; Zurek, E. Decomposition Products of Phosphine Under Pressure: PH2 Stable and Superconducting? ArXiv e-prints 2015, arXiv:1509.05455.

(78) Flores-Livas, J. A.; Amsler, M.; Heil, C.; Sanna, A.; Boeri, L.; Profeta, G.; Wolverton, C.; Goedecker, S.; Gross, E. K. U. Superconductivity in metastable phases of phosphorus-hydride compounds under high pressure. 2015, arXiv:1512.02132.

(79) Ma, Y.; Duan, D.; Li, D.; Liu, Y.; Tian, F.; Huang, X.; Zhao, Z.; Yu, H.; Liu, B.; Cui, T. The unexpected binding and superconductivity in $\mathrm{SbH}_{4}$ at high pressure. 2015, arXiv:1506.03889.

(80) Oganov, A. R.; Glass, C. W. Crystal structure prediction using ab initio evolutionary techniques: Principles and applications. J. Chem. Phys. 2006, 124, 244704. 